\title{
Futuristic Nanomaterials and Composites: Part I
}

\author{
DIRK LEHMHUS, ${ }^{1,2}$ JAMES NJUGUNA ${ }^{3}$ \\ and MURALIDHARAN PARAMSOTHY ${ }^{4,5,6}$
}

\begin{abstract}
1.-ISIS Sensorial Materials Scientific Centre, University of Bremen, Wiener Strasse 12, 28359 Bremen, Germany. 2.-MAPEX Center for Materials and Processes, University of Bremen, Bibliothekstraße 1, 28359 Bremen, Germany. 3.-Centre for Advanced Engineering Materials, School of Engineering, Robert Gordon University, Garthdee House, Garthdee Road, Aberdeen AB10 7QB, Scotland, UK. 4.-School of Science \& Technology, Singapore Institute of Management University (UNISIM), 461 Clementi Road, Singapore 599491, Singapore. 5.-NanoWorld Innovations (NWI), 1 Jalan Mawar, Singapore 368931, Singapore. 6.-e-mail: mpsothy@yahoo.co.uk
\end{abstract}

\section{INTRODUCTION}

The present special topic "Futuristic Nanomaterials and Composites: Part I" captures the structural as well as functional aspects of nanomaterials and nanocomposites that are unique in some specific way when compared to their microscale counterparts. The uniqueness of structural qualities covered includes enhanced fracture toughness and wear resistance, while uniqueness in functional aspects include enhanced thermal conductivity, electrical transport, in addition to nanoscale sensing and characterization capabilities. Synthesis and processing routes leading to the nanostructures involved are also discussed. Inspiration for this special topic is drawn from two conferences: (1) NANOSTRUC 2014: International Conference on Structural Nano Composites held in Madrid (Spain) on 20-21 May 2014 and (2) Nanocomposites III symposium of the TMS 2015 Annual Meeting \& Exhibition held in Orlando, Florida (USA), on 15-19 March 2015. By linking these two events, this joint special topic manifests a trans-atlantic networking in the field of nanocomposites that will be further strengthened in upcoming events of both series.

Even when considering that the number of publications are generally on the rise, analyzing nanocomposite-related data through sources like Web of Science ${ }^{\mathrm{TM}}$ or Sciencedirect ${ }^{\mathrm{TM}}$, it is clear that research on nanostructured materials is still gaining weight. Figure 1 illustrates this situation based on the aforementioned databases and reflects the fact that foci will shift over time. Though the diagrams can offer only a glimpse of the whole field,

Dirk Lehmhus, James Njuguna, and Muralidharan Paramsothy are the guest editors for the Composite Materials Committee of the TMS Structural Materials Division, and coordinators of the topic Futuristic Nanomaterials and Composites: Part I in this issue. they clearly underline that many researchers are currently focusing on graphene and its potential-both structural and functional-for nanocomposite formulation. Not surprisingly, our special topic reflects this trend, containing one article on this topic, complemented by three contributions looking at carbon nanotube (CNT) applications.

As is implied by the significant publication numbers, nanocomposites is a field as wide as materials science in general. Materials that match the nanocomposite definition can be of polymer, ${ }^{1}$ metal $^{2}$ or ceramic matrix. ${ }^{3}$ Their second phase may be either organic or inorganic, including metals, depending on the purpose of the composite design: Dominant among the second phase variants are carbon-based materials like carbon nanotubes $(\mathrm{CNT})^{4}$ or graphene, ${ }^{5}$ not least because of their applicability to optimizing either functional or structural properties, ${ }^{6}$ or both in parallel. An example of the latter is epoxy matrix strengthening in conventional fibre-reinforced composites by CNTs or graphene to simultaneously improve mechanical characteristics and conductivity to provide lightning strike protection in composite planes without weight penalty. ${ }^{7}$ The wide variety of high-performance composites is facilitated by the unique properties that nanoscale structuring provides through phenomena like surface/interface area increase or crystal- and electronic structure-based effects. The general scope extends from improving mechanical strength ${ }^{2,8}$ to optimization of sensing 9,10 and energy conversion/harvesting ${ }^{11,12}$ properties, conductivity, ${ }^{13}$ catalytic performance, ${ }^{14}$ etc., all the way to enhanced energy storage materials ${ }^{15,16}$ and even improved antimicrobial effectiveness. ${ }^{17}$

With adequately covering the whole area being a truly Sisyphean labour, what links the publications in this issue is that they contain 'unique' aspects 

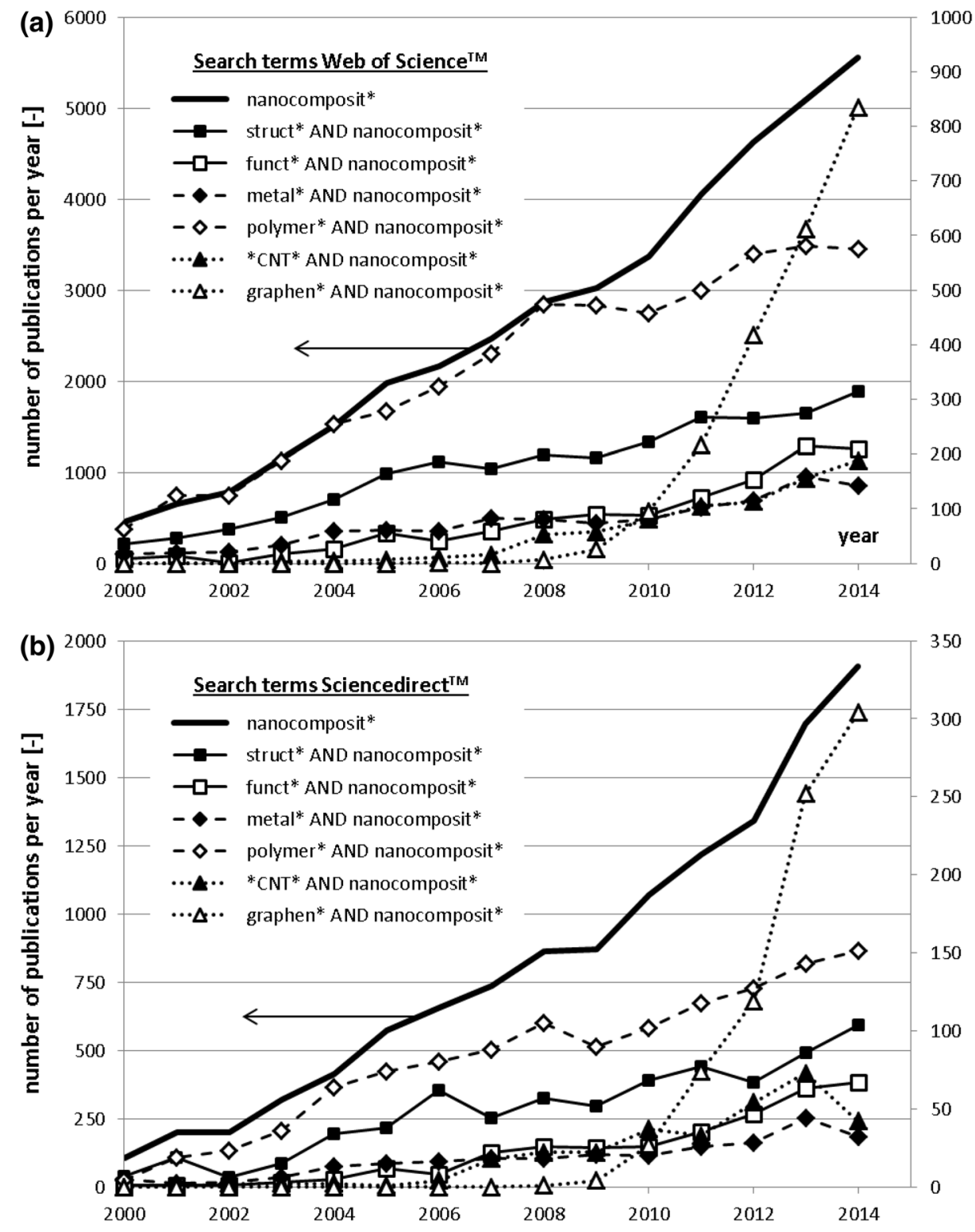

Fig. 1. Publication numbers in the nanocomposite thematic field as found through (a) Web of Science ${ }^{\mathrm{TM}}$ (data retrieved June 14, 2015) and (b) Sciencedirect ${ }^{\mathrm{TM}}$ (data retrieved June 12, 2015) for different search terms and search strategies.

that constitute their immediate relevance concerning development in their respective fields of study. In this JOM topic, they are organized according to their focus in material development: Is it structural (i.e., in a broader sense used here, mechanical) or functional performance that is aimed at? Accordingly, the following sections will first look at contributions addressing the structural issue, and present them briefly, followed by those concentrating on optimized functional performance.

\section{STRUCTURAL ASPECTS}

In the study entitled "Crack Formation in Powder Metallurgy Carbon Nanotube (CNT)/Al Composites during Post Heat-Treatment", by Biao Chen et al. post-heat treatment (PHT) was applied to bulk pure $\mathrm{Al}$ and $\mathrm{CNT} / \mathrm{Al}$ composites fabricated under different processes. It was observed that the nanocomposites consolidated by hot-extrusion formed micro-cracks, but those consolidated by spark plasma sintering (SPS) showed no cracking. A high-temperature SPS process before hot-extrusion was effective in preventing crack formation. The release of residual stress in severe plastic deformed (SPD) materials was responsible for the cracking phenomena during the PHT process. Good particle bonding was essential and effective to suppress cracks for SPD nanocomposite materials in the PHT process.

In the article "Wear Characterization of Carbon Nanotubes Reinforced Acetal Spur, Helical, Bevel and Worm Gears Using TS Universal Test Rig", by 
Samy Yousef et al. CNTs were dispersed into acetal and nanocomposite gears were formed using injection molding. The wear resistances of the gears were measured and the results indicated that the wear resistances of the CNT/acetal gears were increased due to the addition of CNT, especially in the case of the helical, bevel and worm gears.

\section{FUNCTIONAL ASPECTS}

The article "Improved Thermal Conductivity in Carbon Nanotubes-Reinforced Syntactic Foam Achieved by a New Dispersing Technique", by P. Bhat et al., describes the synthesis and characterization of carbon nanotube-reinforced syntactic foams. Carbon nanotubes were dispersed throughout the polymer matrix by growing them on the surface of the glass microballoons which as additives constitute the syntactic foam. Nanotube-grown microballoons improved the thermal conductivity of syntactic foam by $86 \%$ and $92 \%$ (at $50^{\circ} \mathrm{C}$ ) compared to plain and nanotube-mixed syntactic foams, respectively. The improved thermal conductivity as well as the microstructural analysis proved the effectiveness of this new approach for dispersing nano-scale reinforcements in syntactic foams.

In the report on "Nanocalorimetric Characterization of Heterogeneous Nucleation of Rapidly Solidified Bismuth Droplets Embedded in a Zinc Matrix" by Linfang $\mathrm{Li}$ et al. a $\mathrm{Zn}-10 \mathrm{Bi}$ (wt.\%) alloy with Bi droplets in size from micro- to nanometers embedded in the $\mathrm{Zn}$ matrix was prepared. Nanocalorimetry was used to investigate the melting and solidification behavior of the embedded Bi droplets. Nucleation kinetics of rapidly solidified nano-sized $\mathrm{Bi}$ droplets were studied based on the hemispherical cap model of heterogeneous nucleation, and the calculated results revealed the validity of classical heterogeneous nucleation theory in nano-scale at relatively high cooling rates. Due to its high sensitivity and controllable cooling rate, nanocalorimetry is proven as a promising technique to investigate the rapid solidification behavior of embedded nano-sized droplets.

In their article "Structural and Electrical Property of Sol-Gel Derived Nano-Films Lead TitanateBased MIM Capacitor at Different Pb Content", Nurbaya et al. discuss the build-up of metal-insulator-metal capacitors from lead titanate nanofilms created through an adapted spin-coating process on glass substrates. Structural and electrical properties of these electronic components were evaluated and linked to the observed lead acetate levels of 5$25 \mathrm{wt} . \%$, showing most promising functional characteristics at $10 \mathrm{wt} . \%$ and frequencies below $100 \mathrm{kHz}$. Further development towards data storage applications is suggested.

In their contribution titled "Graphite to Graphene via Graphene Oxide: An Overview on Synthesis, Properties, and Applications", D.P. Hansora et al. review the current state of graphene and graphenebased materials in terms of preparation, properties, functionalizations and promising applications. They complement this review with the presentation of a novel, arc-discharge technique for the synthesis of uniform graphene sheets dispersed in water, together with a description of the subsequent processing steps including purification, drying etc., necessary to obtain the final product. Characterization of the latter material using Raman spectroscopy, x-ray diffraction, scanning electron microscopy, and transmission electron microscopy techniques is added.

"Natural ceramic nanotube substrates for surface-enhanced Raman spectroscopy" have been investigated by Vinokurov et al. showing improvements in SERS studies underlined by enhancement factor estimates ranging between 3 and 5 orders of magnitude. The contribution provides details of the synthesis of these materials from halloysite clay nanotubes deposited on aluminium foil and covered by $\mathrm{Au}, \mathrm{Ag}$, and $\mathrm{Cu}$ metal films produced by vacuum evaporation. The possibility of omitting a capping agent during analysis of the $p$-mercaptobenzoic acid used as reference substance opened up by the buildup of the substrates eliminated the respective background peaks from the spectra obtained and led to the aforementioned improvement.

Works by $\mathrm{Li}$ et al. as well as Chen et al. were presented at the TMS 2015 Annual Meeting in the framework of the symposium Nanocomposites III, while the studies by Hansora et al. Nurbaya et al. and Vinokurov et al. formed part of the NANOSTRUC 2014 Conference.

\section{SUMMARY}

Nanomaterials including composites containing nanostructures are currently a dynamic field globally. Traditional composite technologies typically provide composites for singular applications and uses. In contrast, the nanoscale domain is well known for its potential to merge crystal structure (for mechanical properties) and electronic structure (for functional properties) modification, enabling the creation of unique multifunctional nanomaterials. In several ways, such achievements may contribute significantly to sustainability by their enhanced overall performance per discipline, and by enabling the use of a single material to address several functional requirements in parallel, thus reducing the number of product or system components, etc.

The following papers being published under the topic of Futuristic Nanomaterials and Composites: Part I provide excellent details and research on the subject. To download any of the papers, follow the URL http://ink.springer.com/journal/11837/67/12/ page/ 1 to the table of contents page for the December 2015 issue (vol. 67, no. 12).

- "Improved Thermal Conductivity in Carbon Nanotubes-Reinforced Syntactic Foam Achieved by a New Dispersing Technique" P. Bhat, E. Zegeye, A.K. Ghamsari, and E. Woldesenbet 
- "Graphite to Graphene via Graphene Oxide: An Overview on Synthesis, Properties, and Applications" D.P. Hansora, N.G. Shimpi, and S. Mishra

- "Structural and Electrical Property of Sol-Gel Derived Nano- Films Lead Titanate-Based MIM Capacitor at Different Pb Content" Z. Nurbaya, M.H. Wahid, M.D. Rozana, S.A.H. Alrokayan. J.A. Khan, W.C. Gan, W.H.A. Majid, and M. Rusop

- "Surface-Enhanced Raman Spectroscopy Substrates Based on Natural Ceramic Nanotubes" Vladimir A. Vinokurov, Dmitry S. Kopitsyn, Mikhail S. Kotelev, Evgenii V.Ivanov, Yuri M. Lvov, and Andrei A. Novikov

- "Nanocalorimetric Characterization of Heterogeneous Nucleation of Rapidly Solidified Bismuth Droplets Embedded in a Zinc Matrix" Linfang Li, Bingge Zhao, Bin Yang, Quanliang Zhang, Qijie Zhsai, and Yulai Gao

- "Crack Formation in Powder Metallurgy Carbon Nanotube (CNT)/Al Composites during Post Heat-Treatment" Biao Chen, Hisashi Imai, Shufeng Li, Lei Jia, Junko Umeda, and Katsuyoshi Kondoh

- "Wear Characterization of Carbon Nanotubes Reinforced Acetal Spur, Helical, Bevel, and Worm Gears Using TS Universal Test Rig” Samy
Yousef, T.A.Osman, Abdelrahman H. Abdalla, and Gamal A. Zpohdy

\section{REFERENCES}

1. D.R. Paul and L.M. Robeson, Polymer 49, 3187 (2008).

2. S.C. Tjong, Mater. Sci. Eng. R 74, 281 (2013).

3. E. Zapata-Solvas, D. Gómez-García, and A. Domínguez-Rodríguez, J. European Ceram. Soc. 32, 3001 (2012).

4. Z. Spitalskya, D. Tasis, K. Papagelis, and C. Galiotis, Prog. Polymer Sci. 35, 357 (2010).

5. V. Singh, D. Joung, L. Zhai, S. Das, S.I. Khondaker, and S. Seal, Prog. Mater Sci. 56, 1178 (2011).

6. S.C. Tjong, Mater. Sci. Eng. R 53, 73 (2006).

7. M. Fogel, P. Parlevliet, M. Geistbeck, P. Olivier, and É. Dantras, Compos. Sci. Tech. 110, 118 (2015).

8. R.J. Young, I.A. Kinloch, L. Gong, and K.S. Novoselov, Compos. Sci. Tech. 72, 1459 (2012).

9. K. Toda, R. Furue, and S. Hayami, Anal. Chim. Acta 878, 43 (2015).

10. S.K. Vashist and J.H.T. Luong, Carbon 84, 519 (2015).

11. Y. Du, S.Z. Shen, K. Cai, and P.S. Casey, Prog. Polym. Sci. 37,820 (2012).

12. W. Liu, X. Yan, G. Chen, and Z. Ren, Nano Energy 1, 42 (2012).

13. Z. Han and A. Fina, Prog. Polym. Sci. 36, 914 (2011).

14. X. An and J.C. Yu, RSC Adv 1, 1426 (2011).

15. H. Chang and H. Wu, Energy Environ. Sci. 6, 3483 (2013).

16. Q. Wang and L. Zhu, J. Polym. Sci. Part B 49, 1421 (2011).

17. P. Dallas, V.K. Sharma, and R. Zboril, Adv. Colloid Interface Sci. 166, 119 (2015). 